\title{
Optimizing Acoustic Measurements of the Boltzmann Constant ${ }^{1}$ \\ Michael R. Moldover \\ National Institute of Standards and Technology, Gaithersburg, MD 20899-8360, USA
}

(Published in Comptes Rendus Physique 10, 815-827 (2009)

\begin{abstract}
We review the progress in acoustic metrology of gases that has occurred since the 1988 measurement of the universal gas constant $R$ using a spherical acoustic resonator. The advances in understanding resonators and in calculating the thermophysical properties of helium $a b$ initio suggest that today one could determine Boltzmann's constant $k_{\mathrm{B}}$ from acoustic measurements using either helium or argon with a relative uncertainty less than $10^{-6}$.

\section{Résumé}

Progrès dans la détermination de la constante de Boltzmann par voie acoustique L’article récapitule les avancées effectuées en métrologie acoustique dans les gaz depuis la mesure de la constante universelle des gaz $R$ effectuée en 1988 en utilisant un résonateur acoustique sphérique. Des progrès substantiels ont été réalisés, tant pour mieux comprendre le fonctionnement des résonateurs acoustiques que pour calculer $a b$ initio, avec une fiabilité accrue, les caractéristiques thermo-physiques de l'hélium. Ils permettent d'envisager désormais une détermination de la constante de Boltzmann $k_{B}$ par voie acoustique avec une incertitude relative meilleure que $10^{-6}$, en utilisant soit l'argon, soit l'hélium.
\end{abstract}

\section{Introduction.}

The kinetic theory of dilute gases and hydrodynamics relate the average kinetic energy $E$ in one degree of freedom, the speed of sound $u$, and the thermodynamic temperature $T$ :

$$
3 E=\frac{1}{2} m v_{\mathrm{rms}}^{2}=\frac{3}{2} k_{\mathrm{B}} T ; \quad u^{2}=\frac{\gamma}{3} v_{\mathrm{rms}}^{2} \quad .
$$

Here, $v_{\text {rms }}$ is the root mean square speed of a gas molecule, $m$ is the mass of the molecule, $k_{\mathrm{B}}$ is the Boltzmann constant, and $\gamma \equiv C_{\mathrm{p}} / C_{\mathrm{V}}$ is the ratio of the specific heat capacities, which is exactly $5 / 3$ for dilute monatomic gases. The International System of Units assigns the exact value $273.16 \mathrm{~K}$ to the temperature of the triple point of water $T_{\mathrm{TPW}}$. Thus, the Boltzmann constant can be determined by measuring the zero-density limit of the speed of sound of a monatomic gas $u_{0}$ at $T_{\mathrm{TPW}}$. (If the monatomic gas is a mixture of isotopes, $m$ is the mole fraction average of the mass of the mixture.)

The speed of sound has the expansion:

$$
u^{2}(p, T)=\left(\gamma k_{\mathrm{B}} T / m\right)\left(1+\beta_{a}(T) p /(R T)+\gamma_{a}(T) p^{2} /(R T)+\ldots\right),
$$

where $\beta_{\mathrm{a}}$ and $\gamma_{\mathrm{a}}$ are the temperature-dependent acoustic virial coefficients. In 1979, Quinn et al. measured $u^{2}\left(p, T_{\mathrm{TPW}}\right)$ using an argon-filled, cylindrical, acoustical interferometer. [1] In effect,

\footnotetext{
${ }^{1}$ Official contribution of the National Institute of Standards and Technology; not subject to copyright in the United States.
} 
they measured the wavelength of a standing sound wave of known frequency by displacing one end of their interferometer through measured distances. Quinn et al. fitted their data to determine $\beta_{\mathrm{a}}, \gamma_{\mathrm{a}}$, and $k_{\mathrm{B}}$ and they determined $m$ from a chemical and isotopic analysis of their argon. In 1986, CODATA [2] recommended using a value of $k_{\mathrm{B}}$ deduced from the results of Quinn et al. and estimated its relative uncertainty as: $u_{\mathrm{r}}\left(k_{\mathrm{B}}\right)=8.5 \mathrm{ppm}$. (Here and below, all uncertainties are one standard uncertainty and we use "ppm" to denote parts in $10^{6}$.)

In 1988, Moldover et al. measured $u^{2}\left(p, T_{\mathrm{TPW}}\right)$ using an argon-filled spherical cavity. [3] They varied the acoustic frequency and deduced the wavelength of radially symmetric standing waves from the volume of the cavity. The cavity's volume was determined by weighing the mercury required to fill it and by using literature values for the density of mercury. Moldover et al. also fitted their data to determine $\beta_{\mathrm{a}}, \gamma_{\mathrm{a}}$, and $k_{\mathrm{B}}$. In 1998, CODATA recommend using a value of $k_{\mathrm{B}}$ that they deduced from the results of Moldover et al. with the small relative uncertainty $u_{\mathrm{r}}\left(k_{\mathrm{B}}\right)=1.8 \mathrm{ppm}$ and this value is still recommended in 2009. [4]

This article reviews the advances in both understanding and technique that have occurred since the work of Quinn et al. and Moldover et al. Nearly all of these advances were introduced to reduce the uncertainty and complexity of primary acoustic thermometry at temperatures that extend well above and well below $T_{\mathrm{TPW}}$. $[5,6,7,8,9]$ Because primary acoustic thermometry requires the accurate measurement of the zero-pressure limit of speed-of-sound ratios for pure monatomic gases, many thermometry-motivated improvements are applicable to acoustic determinations of $k_{\mathrm{B}}$. Such improvements include:

(1) flowing pure gas through the acoustic cavity to reduce the effects of out-gassing [10],

(2) tuning ducts that allow gas flow through the cavity to reduce the ducts' perturbations to the cavity's acoustic resonance frequencies [11],

(3) minimizing gas contamination by using a bakeable gas-handling system and transducers,

(4) analyzing the gas exiting the resonator via gas chromatography [9],

(5) measuring microwave resonance frequencies of the cavity to determine its thermal expansion and possibly its volume [12],

(6) using a quasi-spherical cavity to retain the advantages of radially-symmetric acoustic modes while splitting the degenerate microwave modes [13],

(7) using removable thermometers and multiple thermometer wells in the cavity's walls to compare the resonator's temperature to $T_{\mathrm{TPW}}$ at the time of use and to detect temperature gradients [9],

(8) using theory to reduce the uncertainties of the thermal conductivity of helium and argon gas and the acoustic virials of helium [8],

(9) using finite element models of the elastic response of the cavity's walls to the acoustic resonances, [14] and (10) accurately modeling acoustic transducers [15].

First, we shall discuss the three phenomena that may dominate the uncertainty of the acoustic measurements needed to re-determine $k_{\mathrm{B}}$ using the spherical cavity method of Moldover et al.:

(1) the thermoacoustic boundary layer,

(2) vibrations of the metal shell enclosing the gas-filled cavity, and

(3) the boundary conditions at the interface between the gas and the walls of an acoustic cavity. 
Then, we discuss the use of ducts, the use of theoretical values of the acoustic virial coefficients, and advances in modeling transducers, acquiring acoustic data, and in thermometry. In addition to these advances that benefit acoustic thermometry, an acoustic re-determination of $k_{\mathrm{B}}$ will require either a measurement of a length change or a volume; we mention several techniques under study for doing this. We conclude by mentioning advances in using cylindrical cavities (the method of Quinn et al.).

\section{Phenomena dominating the uncertainty of acoustic measurements of $\boldsymbol{k}_{\mathrm{B}}$}

At low pressures, the dominant contributions to the uncertainty of $k_{\mathrm{B}}$ originate from the mean free path of the gas and from the thermoacoustic boundary layer. At high pressures, the dominant uncertainties originate from the coupling between the gas-filled cavity and the elastic response of the shell surrounding it and also from fitting the virial coefficients of the gas. Thus, there is an optimum, apparatus-dependent pressure for acoustic measurements of $k_{\mathrm{B}}$. (For completeness, we note that the uncertainties from the frequency-dependence of the speed of sound are negligible under the conditions considered here. [16])

\subsection{The optimum range of pressures}

Figure 1 displays estimates of the pressure-dependent uncertainties of $k_{\mathrm{B}}$ resulting from the thermo-acoustic boundary layer [red band, $2 u\left(\Delta f_{\text {therm }} / f\right)$ ], the vibrations of a spherical shell enclosing a spherical cavity [gray band, $2 u\left(\Delta f_{\text {shell }} / f\right)$ ], and the thermal accommodation length $l_{\text {a }}$ [black dashed black line, $2 u\left(l_{a} / a\right)$ ]. These estimates assume that the shell is made of type 316 stainless steel and it has an inner radius $a=5 \mathrm{~cm}$ and an outer radius $b=8 \mathrm{~cm}$. A shell of these dimensions can be fabricated and thermostatted easily; however, the author is not aware that such a thick-walled shell has ever been used for gas metrology. Because $u\left(\Delta f_{\text {shell }} / f\right)$ varies as $p$, $u\left(\Delta f_{\text {therm }} / f\right)$ varies as $p^{-1 / 2}$, and $u\left(l_{\mathrm{a}} / a\right)$ varies a $p^{-1}$, there is an optimum range of pressure for the measurement of $k_{\mathrm{B}}$ which occurs near $1 \mathrm{MPa}$ for helium and $0.3 \mathrm{MPa}$ for argon. In this range,
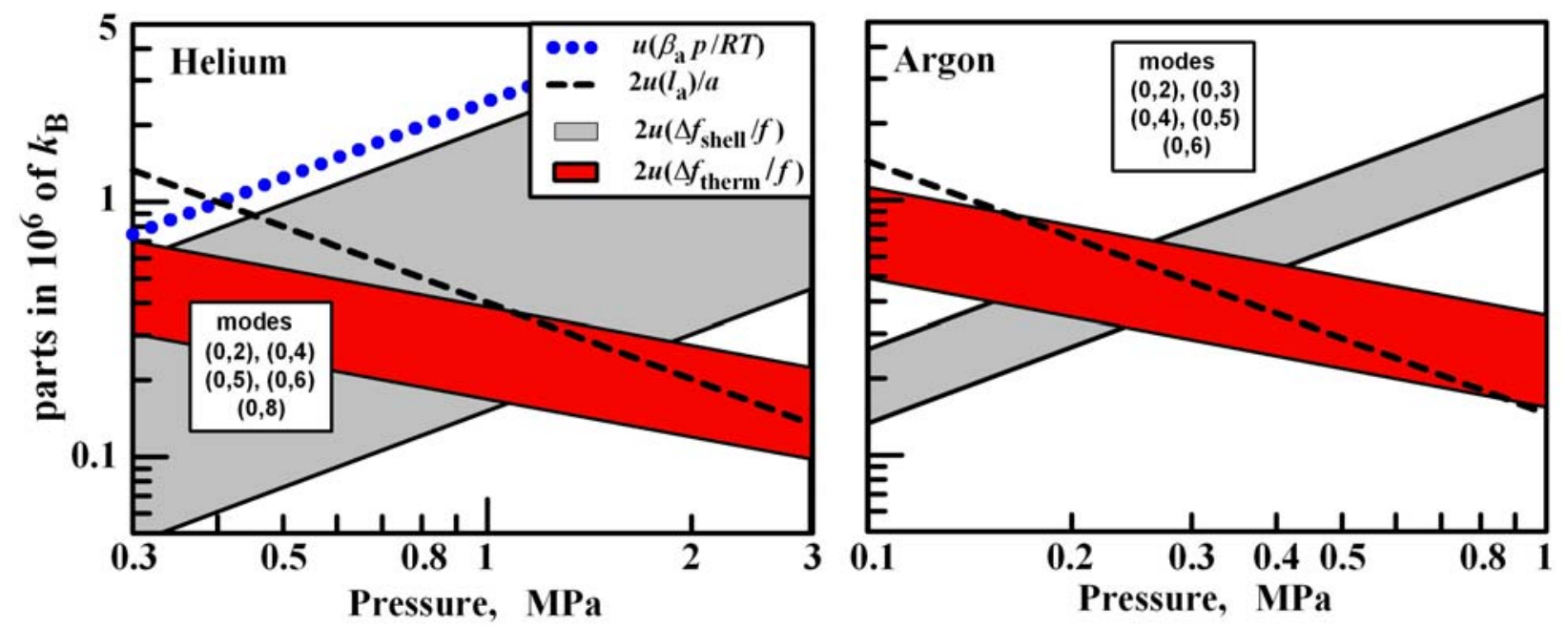

Fig. 1. Contributions to the uncertainty of $k_{\mathrm{B}}$ from the thermoacoustic boundary layer $2 u\left(\Delta f_{\text {therm }} / f\right)$, shell vibrations $2 u\left(\Delta f_{\text {shell }} / f\right)$ and the thermal accommodation coefficient $2 u\left(l_{\mathrm{a}}\right) / a$ for helium and argon. The dotted blue line represents the uncertainty of the calculated speed of sound of helium from the uncertainty of its second acoustic virial coefficient $\beta_{\mathrm{a}}$. In $1988 \beta_{\mathrm{a}}$ was treated as a fitting parameter. 
each of the plotted uncertainties is on the order of $0.5 \times 10^{-6} k_{\mathrm{B}}$. First, we consider $u\left(\Delta f_{\text {therm }} / f\right)$ and $u\left(\Delta f_{\text {shell }} / f\right)$; then we discuss the uncertainty from the gas-shell interface including $u\left(l_{\mathrm{a}} / a\right)$.

\subsection{Thermoacoustic Boundary Layer}

During each acoustic cycle, heat exchange between the gas and the shell results in a thermoacoustic boundary layer in the gas that is characterized by an exponential decay length $\delta_{\text {therm }}=\left[\lambda /\left(\rho C_{\mathrm{p}} \pi f\right)\right]^{1 / 2}$. Here $\lambda$ is the thermal conductivity of the gas, $\rho$ is its density, and $C_{\mathrm{p}}$ is the constant-pressure heat capacity which is exactly $5 R / 2$ for monatomic gases in the limit of zero density.) For the radially-symmetric acoustic modes, the boundary layer contributions to the real and the imaginary (half-width) parts of the resonance frequencies are:

$$
\left(\Delta f_{\text {therm }}+i g_{\text {therm }}\right) / f=(-1+i)(\gamma-1) \delta_{\mathrm{t}} /(2 a)
$$

Thus, $\Delta f_{\text {therm }}$ and $g_{\text {therm }}$ are equal and both increase at low pressures $p^{-1 / 2}$. (See Fig. 2, left.)

At low pressures at $T_{\mathrm{TPW}}$, the uncertainty of the thermal conductivity $u(\lambda)$ dominates the uncertainty of $\Delta f_{\text {therm. }}$. The most accurate, zero-pressure values of the thermal conductivity of helium are those calculated $a b$ initio from quantum mechanics and statistical mechanics. $[17,18]$. In the limit of zero density at $T_{\mathrm{TPW}}$, the most accurate value of the thermal conductivity of argon $\lambda_{\mathrm{Ar}}$ is obtained by combining the calculated value of the Prandtl number $\operatorname{Pr} \equiv C_{\mathrm{p}} \eta / \lambda$, the calculated value of the viscosity of helium $\eta_{\mathrm{He}}$, and the measurements by May et al of the ratio $\eta_{\mathrm{Ar}} / \eta_{\mathrm{He}} \equiv$ (viscosity of argon)/(viscosity of helium). [19] In Ref. [19], the relative uncertainty of the viscosity ratio measurement was $u_{\mathrm{r}}\left(\eta_{\mathrm{Ar}} / \eta_{\mathrm{He}}\right)=0.00011$ and the uncertainty of the Prandtl
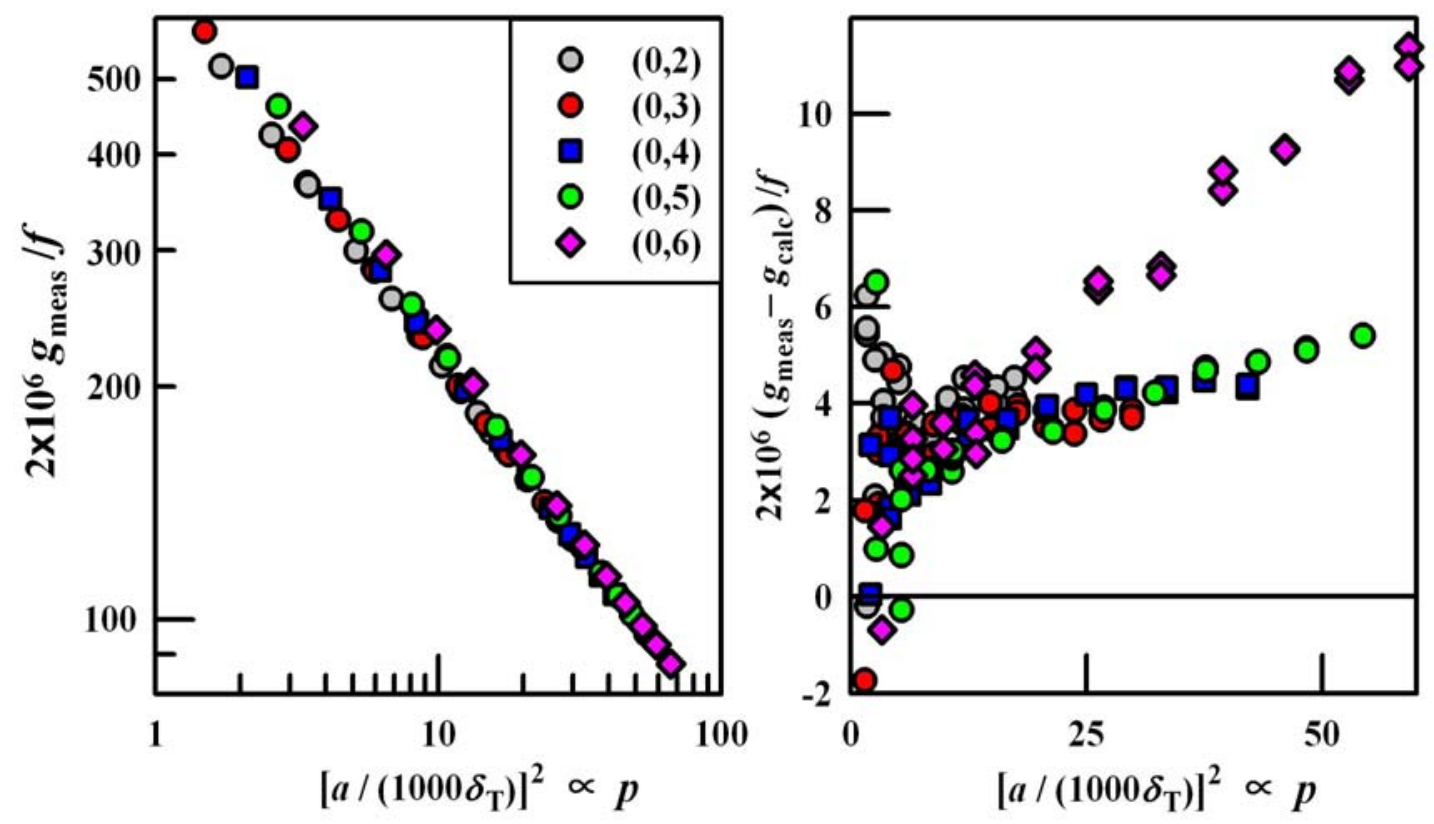

Fig 2. Left: half-widths of 5 radial acoustic modes of an argon-filled cavity measured during the 1988 determination of $k_{\mathrm{B}}$. [3] The horizontal axis is proportional to pressure. Right: differences between measured and calculated half-widths. Linear extrapolations of the differences to zeropressure yield intercepts that are equivalent to 2 to $3 \mathrm{ppm}$ of $k_{\mathrm{B}}$. Note: The values of $g_{\text {calc }}$ include a small contribution from the attenuation of sound throughout the volume of the cavity. 


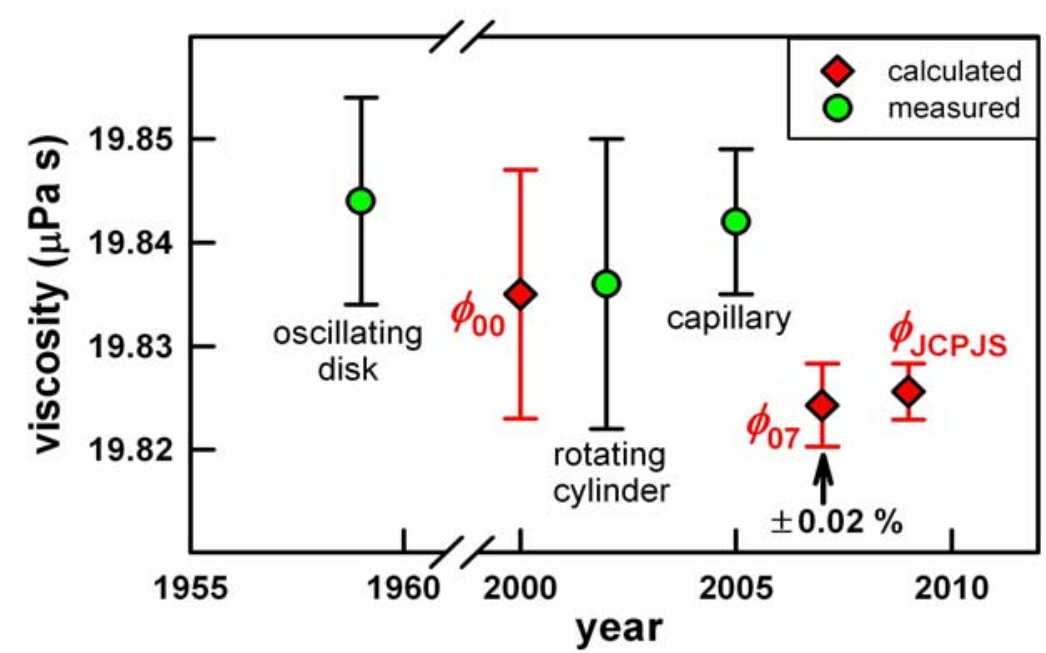

Fig. 3 Accurate determinations of the viscosity of helium in the limit of zero pressure at $298.15 \mathrm{~K}$. Data sources: oscillating disk [20]; rotating cylinder [21]; capillary [22]; $\phi_{00}[23] ; \phi_{07}[17] ; \phi_{\mathrm{JCPJS}}[25]$.

number was $u(P r)=0.00004$. Equation (37) of [17] provides the uncertainty of the calculated value $u_{\mathrm{r}}\left(\eta_{\mathrm{He}}\right)=0.00022$. The sum in quadrature of these three uncertainties is $u_{\mathrm{r}}\left(\lambda_{\mathrm{Ar}}\right)=0.00025$, and we used this value for argon in Fig. 1.

Our value $u_{\mathrm{r}}\left(\lambda_{\mathrm{Ar}}\right)=0.00025$ is only $3 / 10$ of the uncertainty $u_{\mathrm{r}}\left(\lambda_{\mathrm{Ar}}\right)=0.00084$ reported by May et al. [19] The two different uncertainty estimates of $u_{\mathrm{r}}\left(\lambda_{\mathrm{Ar}}\right)$ results from two different estimates of the uncertainty of $\eta_{\mathrm{He}}\left(p=0, T_{\mathrm{TPW}}\right)$. Here, we refer to Fig. 3 to discuss $\eta_{\mathrm{He}}$ at 298.15 K where accurate viscosity measurements were made. We argue for the smaller uncertainty estimate at $298.15 \mathrm{~K}$ and use it at $T_{\text {TPW }}$.

In 2006, May et al. estimated $u\left(\eta_{\mathrm{He}}\right)$ as the difference between the best available measured and calculated values $\eta_{\mathrm{He}}(p=0,298.15 \mathrm{~K})$. They considered the oscillating disk measurements by Kestin and Leidenfrost [20]; the rotating cylinder measurements by Evers et al [21]; and Berg's quartz capillary measurements [22]. They also considered the value calculated ab initio in 2000 using the helium-helium potential $\phi_{00}$ [23] and the value published in 2007 calculated with the potential $\phi_{07}$ [17]. After May et al. [19] published their conclusions, Jeziorska et al. [24] developed a more accurate potential $\phi_{\text {JCPJs }}$ that Mehl [25] used to calculate the values $\eta_{\mathrm{He}}(p=0,298.15 \mathrm{~K})=(19.8269 \pm 0.0002) \mu \mathrm{Pa} \cdot \mathrm{s}$ and $(19.8242 \pm 0.0002) \mu \mathrm{Pa} \cdot \mathrm{s}$, where the quoted uncertainty only accounts for the uncertainty of the helium-helium potential $\phi_{\text {JCPJS. }}$ The larger of Mehl's two values uses the atomic mass of helium; the smaller uses the nuclear mass. Because we do not know which mass is correct, we plotted at 2009 in Fig. 3 the average of Mehl's two values and used the difference between his values $(0.0027 \mu \mathrm{Pa} \cdot \mathrm{s})$ as the uncertainty bar. The relative uncertainty $u_{\mathrm{r}}\left(\eta_{\mathrm{He}}\right)$ is only 0.00013 .

In private communications, R. F. Berg (2009, NIST) indicated that some Type B uncertainties were not included in his uncertainty budget for the capillary measurement. [22] In particular, Berg used only one quartz capillary and determined its average internal diameter by weighing a mercury drop that he forced into the capillary. The diameter was not measured as a function of length. The manufacturer's claim concerning the roundness of the capillary's cross sections was verified only indirectly by measuring the external diameter and by the consistency of flow measurements at large Dean numbers. Berg fitted his helium data with a momentumaccommodation coefficient $h_{\text {mometum }}$ to eliminate its unphysical pressure-dependence. The best- 
fit values of $h_{\text {mometum }}$ were 1.09, 1.19 and 1.14 during the years 2001, 2002 and 2003, respectively. Berg does not know why $h_{\text {mometum }}$ changed; however, his values are closer to 1 than previous measurements of helium in glass capillaries. [26]

We illustrate the importance of the thermoacoustic boundary layer in Fig. 2 by replotting the resonance half-widths $g_{\text {meas }}$ that were measured during the 1988 re-determination of $k_{\mathrm{B}}$. [3] In the pressure range $25 \mathrm{kPa}<p<500 \mathrm{kPa}$, between $80 \%$ and $99 \%$ of $g_{\text {meas }}$ was generated in the thermoacoustic boundary layer. The calculated half-widths $g_{\text {calc }}$ used the thermophysical properties of argon, the geometry of the resonator (including crevices), and the attenuation of sound throughout the volume of the resonator. As the pressure approaches zero, the values of $2 \Delta g / f \equiv 2\left(g_{\text {meas }}-g_{\text {calc }}\right) / f$ approach $2.5 \times 10^{-6}$ for all of the modes studied. Today, we still do not know why $\lim _{p \rightarrow 0}(2 \Delta g / f)>0$ and we consider the average limiting value $\left(2.5 \times 10^{-6}\right)$ to be a measure of our imperfect understanding of the acoustic resonator. In 1988, this limiting value was acceptable because it was only 1.5 times the uncertainty claimed for $k_{\mathrm{B}}: u_{\mathrm{r}}\left(k_{\mathrm{B}}\right)=1.7 \times 10^{-6}$. If an acoustic resonance technique were used to re-determine $k_{\mathrm{B}}$ today, we would expect a much smaller claimed uncertainty. We would also expect correspondingly smaller values of $\lim _{p \rightarrow 0}(2 \Delta g / f)$ to confirm the claim of improved understanding of the acoustic resonator.

In summary, the uncertainties of the calculated values of $\Delta f_{\text {therm }} / f$ and $g_{\text {calc }} / f$ have been substantially reduced since the 1988 determination of $k_{\mathrm{B}}$ because the uncertainties of the transport properties of helium and argon have been greatly reduced. The reduction in $u\left(\eta_{\mathrm{He}}\right)$ at $p=0$ and $298.15 \mathrm{~K}$ is illustrated in Fig. 3. Under the same conditions, the uncertainty of the Prandtl number $u(P r)=0.00004$; therefore, $u_{\mathrm{r}}\left(\lambda_{\mathrm{He}}\right)$ is only slightly greater than $u_{\mathrm{r}}\left(\eta_{\mathrm{He}}\right)$. [19]

\subsection{Coupling between gas modes and shell vibrations}

The effects of shell motion on the gas resonances have been discussed by Mehl. [27] When a radially symmetric gas resonance is not too close to the breathing resonance of an isotropic spherical shell, the frequency of the gas resonance is shifted by

$$
\frac{\Delta f_{\text {shell }}}{f}=\frac{-\gamma p \chi_{\mathrm{s}, \mathrm{i}} / 3}{1-\left(f / f_{\text {breathing }}\right)^{2}} \text {, }
$$

where $\chi_{\mathrm{s}, \mathrm{i}} \equiv(3 / a)\left(d a / d p_{\mathrm{i}}\right)$ is the shell's compliance to internal pressure $p_{\mathrm{i}}$. To prepare Fig. 1 , we assumed that $\chi_{\mathrm{s}, \mathrm{i}}$ could be determined with an uncertainty of $10 \%$. To support this uncertainty estimate, we recall that Moldover et al. [3] determined the static compliance of the shell in three independent ways: (1) theoretically, based on equations for a thick, spherical shell and published values for the elastic properties of 316 stainless-steel; (2) experimentally, by measuring acoustic resonance frequency as a function of changes in the external pressure of the resonator; and, (3) experimentally, by measuring the expansion of the shell under applied internal pressure while the shell was filled with mercury. (Today, one might also determine compliances by measuring the effect of internal or external pressure on microwave resonances of the cavity.) The compliance determined by method (2) was $6 \%$ greater than that determined by method (1); however, this was within the combined uncertainties. The compliance determined by methods (1) and (3) agree within tighter uncertainty bounds [2 \% for method (1) and $1 \%$ for method (3)]. Method (1) was also used to estimate the frequency of the breathing mode of the shell $f_{\text {breathing }} \approx 13.58 \mathrm{kHz}$. A shell resonance was found near $13.2 \mathrm{kHz}$. The difference between $13.58 \mathrm{kHz}$ and $13.2 \mathrm{kHz}$ is equivalent to a $1.5 \%$ difference in the compliance. 
In the left panel of Fig. 1, the gray band labeled $u\left(\Delta f_{\text {shell }} / f\right)$ spans the expected uncertainties in $k_{\mathrm{B}}$ resulting from a $10 \%$ uncertainty of $\chi_{\mathrm{s}, \mathrm{i}}$. For helium, the band spans estimates for the $(0,2)$, $(0,4),(0,5),(0,6)$, and $(0,8)$ radially-symmetric acoustic modes. The $(0,3)$ mode cannot be used for helium gas metrology because its resonance frequency is close to $f_{\text {breathing; }}$ therefore, it is strongly perturbed by the shell's motion. The $(0,7)$ mode cannot be used for gas metrology because it is nearly coincident with the $(13,2)$ mode, as shown in Fig. 8 of [3]. Because the shell's compliance and its uncertainty both scale as $\left[1-\left(f / f_{\text {breathing }}\right)^{2}\right]^{-1}$, the smallest values of $u\left(\Delta f_{\text {shell }} / f\right)$ for helium will occur for the $(0,6)$ and $(0,8)$ modes. By accident, the resonance frequencies of the $(0,6)$ and $(0,8)$ modes occur within $1 \%$ of the frequencies of non-radial shell modes predicted for a steel shell with $a=50 \mathrm{~mm}$ and wall $30 \mathrm{~mm}$ thick. The $(0,6)$ or $(0,8)$ mode can be used re-determine $k_{\mathrm{B}}$ if the shell's thickness is slightly adjusted to increase the separation between these modes and the non-radial modes of the shell.

The gray band in the right panel of Fig. 1 spans the uncertainties expected for the $(0,2)$ through $(0,6)$ radially-symmetric argon acoustic modes; the expected uncertainties increase monotonically with frequency. The $(0,8)$ mode cannot be used because its frequency will be close to $f_{\text {breathing }}$ and the $(0,7)$ mode cannot be used because it is nearly coincident with the $(13,2)$ mode, as mentioned above.

Two research groups have studied the interaction of the radial acoustic modes with shell modes by continuously varying the speed of sound. Pitre et al. [28] varied the speed of sound by changing the temperature over a very wide range. Gavioso et al. [29] filled the cavity with helium and then continuously diluted it with argon while maintaining the pressure constant. Both groups were surprised to observe interactions between the radially-symmetric acoustic modes and several modes of the shell at frequencies well away from $f_{\text {breathing. These observations }}$ stimulated J. B. Mehl and M. R. Moldover [30] to model the vibrations of shells that depart from spherical symmetry. They modeled a shell that was bound by concentric spherical surfaces and was supported by a stiff connection to a large mass. They also modeled shells with spherical inner surfaces and outer surfaces that had large bosses and/or flanges similar to those on the resonators used for acoustic thermometry. [5,6,7,8,9] Consistent with the observations of Pitre et al. and Gavioso et al., the models predict that radial acoustic modes do couple to non-radial shell modes when the spherical symmetry is broken, either by the shape of the outer surface or by a stiff support. Optimizing an acoustic resonator to measure $k_{\mathrm{B}}$ will minimize its departures from spherical symmetry.

Because $\Delta f_{\text {shell }} \propto p$, it is not essential to know the compliance of the shell accurately. Instead, one can treat the linear term in the pressure expansion $u^{2}\left(p, T_{\mathrm{TPW}}\right)$ as a separate fitting parameter for each mode instead of the mode-independent thermodynamic quantity $\beta_{\mathrm{a}} / R T$. In doing so, a test of understanding is abandoned. Yet another option is to reduce the compliance of a shell (and its uncertainty) by manufacturing it out of a stiffer material. A beryllium shell will have $\sim 1 / 2$ the compliance of a steel shell with the same dimensions. However, the complex metallurgy and toxicity of beryllium might create new problems.

\subsection{Gas-Solid Boundary Conditions}

\subsubsection{Thermal accommodation coefficient model}

Ewing et al. [31] discussed the acoustic consequences of the kinetic theory prediction that a temperature jump occurs at a gas-solid interface when heat is transferred across the interface. 
[32] They concluded that the temperature jump increases the resonance frequencies and leaves the half-widths unchanged. The frequency increase is $\Delta f / f=(\gamma-1) l_{\mathrm{a}} / a$, where $l_{\mathrm{a}}$ is the thermal accommodation length, which for a monatomic gas is given by

$$
l_{a}=(\lambda / p) \sqrt{\pi m T /\left(2 k_{\mathrm{B}}\right)}(2-h) / h \quad .
$$

Here, $\lambda$ is the thermal conductivity, $m$ is the mass of an atom, and $h$ is the thermal accommodation coefficient. (If $h=1, l_{\mathrm{a}}$ equals 1.8 times the mean free path. For argon at $T_{\mathrm{TPW}}$, $100 \mathrm{kPa}$, and $h=1, l_{\mathrm{a}}=118 \mathrm{~nm}$.) The coefficient $h$ accounts for the fraction of the gas molecules incident on the solid that are reflected or re-emitted from the solid with the kinetic energy expected from the solid's temperature. Thus $h$ might depend upon the gas, the temperature, and the microscopic conditions of the surface (e.g. oxidized or covered with an oil film). The temperature jump adds a $p^{-1}$ term to the polynomial expansion of $f^{2}(p, T=$ const) where $f$ is the measured acoustic resonance frequency on an isotherm. Ewing et al. [31] included a $p^{-1}$ term in a fit to their measurements of $f^{2}\left(p, T_{\mathrm{TPW}}\right)$ for an argon-filled, aluminum-walled cavity and found $h=(0.84 \pm 0.05)$. For an argon-filled, steel-walled cavity, Moldover et al. [3] found $h=(0.93 \pm$ $0.07)$ at $T_{\text {TPW }}$. We use this experience as a guide to assume that $u(h)=0.05$. The dashed black lines in Fig. 1 display how $u(h)$ propagates into an uncertainty of $2 u\left(l_{\mathrm{a}}\right) / a$. (The factor of 2 is included because $u\left(k_{\mathrm{B}}\right)$ is proportional to the square of the acoustic radius of the resonator.) The locations of dashed lines on Fig. 1 are insensitive to $h$ as long as $h$ is on the order of 1 . If $l_{\mathrm{a}}, \delta_{\mathrm{t}}$, and $\delta_{\text {viscosity }}$ were the only lengths relevant to the gas-solid interactions, measurements of $f(p, T)$ at low pressures could reduce $u(h)$ below 0.05 . However, the measurements are not easy because the signal-to-noise ratio decreases as $p^{-2}$ below an apparatus-dependent pressure (100 kPa in [3]). Also, $Q$ decreases with pressure; therefore; values $f_{\text {fit }}$ and $Q_{\text {fit }}$ that result from fitting the usual resonance function have to be corrected. For these corrections, Gillis et al. [33] deduced the formulas

$$
\frac{f_{\text {corr }}-f_{\text {fit }}}{f_{\text {fit }}} \approx-\frac{1}{8} Q_{\text {surf }}^{-2}+\frac{1}{4} Q_{\text {vol }}^{-2}+\frac{1}{8} Q_{\text {surf }}^{-1} Q_{\text {vol }}^{-1}-\frac{1}{8} Q_{\text {surf }}^{-3} \text { and } Q_{\text {corr }}^{-1}-Q_{\text {fit }}^{-1} \approx-\frac{1}{4} Q_{\text {surf }}^{-2}-(0.263) Q_{\text {surf }}^{-1} Q_{\text {vol }}^{-1}+\frac{1}{4} Q_{\text {surf }}^{-3}
$$

that account for boundary losses via $Q_{\text {surf }}$ and the attenuation of sound in the volume via $Q_{\text {vol }}$. The background terms in the resonance formula also generate corrections of order $Q^{2}$.

\subsubsection{Corrugated surface model}

The kinetic theory calculation that relates the accommodation coefficient to the accommodation length assumes that the solid is a perfectly smooth plane; thus, the mean free path is the only microscopic length in the calculation. Machined metal surfaces are rough and are characterized by many length scales. Thus, the real gas-solid boundary conditions will be more complicated that those assumed by Ewing et al. and by the 1988 re-determination of $k_{\mathrm{B}}$.

Using numerical methods, Mehl [34] estimated $\Delta f_{\text {corrugation, the perturbation to the acoustic }}$ resonance frequencies cause by a corrugation model of a rough surface. The model surface had the profile $z=Z \cos q x$, where $\lambda_{\mathrm{s}}=2 \pi / q$ is the spatial wavelength and $Z$ is the amplitude of the corrugation. In the limit of $\delta_{\mathrm{t}} / \lambda_{\mathrm{s}}>>1$, the regions between the maxima of the surface profile form a nearly isothermal volume whose magnitude is of order $Z \times A_{\mathrm{p}}$, where $A_{\mathrm{p}}$ is the projected area. In the absence of corrugations, the isothermal volume is $\delta_{\mathrm{t}} A_{\mathrm{p}}$. Thus, the perturbation from the thermoacoustic boundary layer is increased by the factor: $1+O\left(Z / \delta_{\mathrm{t}}\right)$. To estimate $Z / \delta_{\mathrm{t}}$, we consider typical measurement conditions [argon; $a=50 \mathrm{~mm} ;(0,4)$ mode; $0.3 \mathrm{MPa} ; \delta_{\mathrm{t}}=$ $13 \times 10^{-6} \mathrm{~m}$ ] and a hypothetical steel surface that has been finished with a surface grinder that 
generated a surface finish with a root-mean-square roughness of order $0.2 \times 10^{-6} \mathrm{~m}$. For this example, the corrugations increase the frequency shift and half-width perturbations by $O(1.5 \%)$ and this increase varies with pressure as $p^{1 / 2}$. A convenient way to express this result is:

$$
\frac{\Delta f_{\text {corrugation }}}{f}=O\left(1.4 \times 10^{-6} \sqrt{\frac{p}{0.3 \mathrm{MPa}} \frac{f_{(0,4)}}{f}}\right) .
$$

In the same limit $\delta_{\mathrm{t}} / \lambda_{\mathrm{s}}>>1$, Mehl found smaller values of $\Delta g_{\text {corrugation }} / f$.

We recommend carefully polishing the interior surface of the cavity. (For the 1988 redetermination of $k_{\mathrm{B}}$, the final polishing of the steel used $\alpha$ - and $\gamma$-alumina pastes with 0.3 and $0.05 \mu \mathrm{m}$ particle sizes.) Because surface roughness occurs on many length scales, $\Delta f_{\text {roughness }} / f$ will surely have a more complicated pressure dependence than either $p^{-1}$ predicted by the thermal accommodation coefficient model or $(p / f)^{1 / 2}$ inferred from the corrugated surface model.

\section{Ducts that Admit Gas into a Cavity}

During the 1988 determination of $k_{\mathrm{B}}$, a single duct was used to conduct argon into and out of the resonator. [3] A manually operated valve had been built into the duct. While the acoustic resonance frequencies were measured, this valve was maintained in its closed position where the end of its specially fabricated stem was flush with the surface of the spherical cavity. (See Fig. 3 of [3].) This arrangement was advantageous because the closed valve made the smallest possible perturbation to the acoustic resonance frequencies. However, the valve's vacuum-tight seal was made by a small fluoropolymer O-ring. This O-ring and two other O-rings that sealed the acoustic transducers in their places were potential sources of gas contamination. To minimize contamination, the apparatus was flushed with pure argon numerous times before it was used. After flushing, no contamination was detected at $T_{\text {TPW }}$; however, progressive contamination was observed when the apparatus was operated at $30{ }^{\circ} \mathrm{C}$. [5]

Ripple et al. rebuilt the resonator used in the 1988 determination of $k_{\mathrm{B}}$ for acoustic thermometry at high temperatures. [35] They did not re-polish the interior surface of the resonator; however, they replaced the single fill duct and valve with two long, $1.5 \mathrm{~mm}$-bore, ducts, one to serve as a gas inlet and the other as an outlet. At the wall of the spherical shell, these ducts opened into a $1 \mathrm{~cm}^{3}$ volume that was connected to the cavity by a short duct of length $a / 10$ as suggested by [11]. These ducts made small, predictable perturbations to the radial acoustic modes and allowed a small, steady flow of argon to remove hydrogen that out-gassed from the hot steel walls of the cavity. A measure of the ducts' performance is $\Delta g / f$, where the excess half-width $\Delta g \equiv g_{\text {meas }}-g_{\text {calc }}$ is the difference between the measured half-width and that calculated from the thermophysical properties of argon and the geometry of the resonator, including its ducts. [9] Typically, $\Delta g / f$ varied from $\sim 10 \mathrm{ppm}$ at high densities to a zero-pressure limit near $2 \mathrm{ppm}$. The zero-pressure limit of $\Delta g / f$ was independent of mode and temperature demonstrating that the effects of the ducts cancelled out of the determination of the thermodynamic temperature $T$. This behavior of $\Delta g / f$ is consistent with that observed at $T_{\text {TPW }}$ during the 1988 re-determination of $k_{\mathrm{B}}$ (Fig. 2) when a valve at the cavity closed a single duct. (Note: Fig. 2 is a plot of $2 \times \Delta g / f$.)

In contrast with Ripple et al, Pitre et al [8] and, independently, Benedetto et al. [7] used ducts with lengths equal to their respective cavities' radii $a$. These ducts led from the cavities to the volume inside pressure vessels surrounding the cavities. Both groups found larger values of $\Delta g / f$ than they could explain, particularly for the $(0,2)$ mode. After their measurements were 
completed, they found evidence that the mating surfaces of the hemispheres were not sufficiently flat to avoid a crevice at the equatorial joint. [28] (A crevice will increase $\Delta g / f$.) Because, the zero-pressure limits of $\Delta g / f$ in [7] and [8] were independent of temperature for each mode, the acoustic thermometry was successful. (In [8] the same limits were found for helium and argon.) However, a re-determination of $k_{\mathrm{B}}$ cannot rely on temperature-independence; the perturbations from imperfect spherical geometry (e.g. ducts, crevices, acoustic and microwave transducers) must be accurately known.

In this context, the acoustic admittance of ducts has been measured using three-port couplers. [36,37] A three-port coupler is a small, sealed, gas-filled volume. One port contains a sound source; a second port contains a sound detector, and the third port is the test port. One measures the complex frequency-dependent acoustic pressure while the test port holds the object under study. Often, the object under study is replaced with an admittance standard (e.g. a solid metal wall that has a calculable admittance) and the acoustic pressure is measured again. The complex ratio of the acoustic pressures is used to deduce the complex ratio of the acoustic admittance of the test object to that of the admittance standard. Lin et al. [37] were unable to obtain satisfactory reproducibility following the usual practice. Instead, they adopted an unusual alternative; they mounted a $1.4 \mathrm{~m}$ long duct in the test port and measured the admittance changes after successive lengths of duct were cut off its open end. (The duct was open to the room at the end opposite the coupler.) The measured admittance of the1.4 m long duct agreed with a model calculation within the measurement's uncertainty of 1\%. For ducts $10 \mathrm{~cm}$ and $20 \mathrm{~cm}$ long, the measured admittance differed from a simple model by as much as $10 \%$ of the admittance. The frequency dependence of the differences resembled that of the acoustic resonances in the duct. This suggests that the sound reflected from the open end underwent a phase shift that was not included in their model. (Note: a $5 \mathrm{~cm}$-long duct with a $1.4 \mathrm{~mm}$ bore is predicted to perturb the $(0,2)$ mode of a 5 -cm radius sphere filled with argon at $0.3 \mathrm{MPa}$ by $(\Delta f+i g) / f=(0.9+1.1 i) \times 10^{-6}$ and the $(0,5)$ mode by $(\Delta f+i g) / f=(-0.8+0.7 i) \times 10^{-6}$. As the pressure is reduced, the predicted perturbations increase approximately as $p^{-1 / 2}$.) The disagreement between measurement and theory reported by Lin et al. for short ducts is much smaller than that reported by Pitre et al. [8] and Benedetto et al. [7].

From these observations, we conclude that an optimized measurement of $k_{\mathrm{B}}$ will certainly require mounting ducts in a way that avoids crevices and might require some combination of three options to reduce the uncertainty of the perturbations. The options are: (1) narrower or longer ducts, (2) systematic measurements of the admittance of the ducts actually used, or (3) a more accurate theory for ducts.

\section{Theoretical values of the acoustic virial coefficients.}

As mentioned in the Introduction, the polynomial expansion of $u^{2}(p, T)$ includes the term $\beta_{\mathrm{a}} p /(R T)$. The value of $\beta_{\mathrm{a}}\left(T_{\mathrm{TPW}}\right)$ for helium calculated $a b$ initio is more accurate than any measurement $\left[\left(u_{\mathrm{r}}\left(\beta_{\mathrm{a}}\right)=0.00084\right.\right.$ in [17] at $\left.T_{\mathrm{TPW}}\right]$ and its uncertainty will decrease. [25] The dotted blue line in Fig. 1 for helium displays the pressure-dependent ratio $\mathrm{u}\left(\beta_{\mathrm{a}}\right) p /(R T)$ at $T_{\mathrm{TPW}}$. This ratio is comparable to the uncertainty of the correction for the breathing mode of the shell. Therefore, comparing the $a b$ initio value of $\beta_{\mathrm{a}}\left(T_{\mathrm{TPW}}\right)$ to the values of $\beta_{\mathrm{a}}\left(T_{\mathrm{TPW}}\right)$ determined by fitting the data for various modes will be a useful test of the understanding of a helium-filled spherical acoustic resonator. 


\section{Modeling acoustic transducers}

Recently, Guianvarc'h et al. [15] studied condenser microphones similar to those used in several acoustic thermometers, in the 1988 re-determination of $k_{\mathrm{B}}$, and in recent experiments directed towards re-determining $k_{\mathrm{B}}$. Guianvarc'h et al. accurately calculated and measured the microphone's characteristics as functions of frequency and pressure for both helium and argon and used their results to calculate the perturbations to the resonance frequencies of the radial acoustic modes of a gas in a spherical cavity. They concluded that the simple approximation ( $\Delta f_{\text {microphone }} / f=-0.8 \times 10^{-6} \mathrm{p} / \mathrm{MPa}$ ) used for the 1988 determination of $k_{\mathrm{B}}$ was satisfactory. (The 1988 measurements used argon in a cavity with $a=89 \mathrm{~mm}$.) However, such a simple approximation is not suitable for re-determining $k_{\mathrm{B}}$ using, for example, the $(0,4)$ and higher modes of a helium-filled cavity with $a=50 \mathrm{~mm}$, because $\Delta f_{\text {microphone }} / f$ has complex dependences on frequency, pressure, and the gas in a wide region spanning the microphone's resonance near $50 \mathrm{kHz}$. [The $(0,6)$ mode of helium occurs at $53 \mathrm{kHz}$.] Even the $(0,2)$ and $(0,3)$ modes of a resonator with $a=50 \mathrm{~mm}$ are problematic because $\Delta f_{\text {microphone }} / f \propto a^{-3}$ at low frequencies.

\section{Remarks on acquiring data and on thermometry}

In 1988, the statistical uncertainties of the acoustic measurements contributed $0.68 \mathrm{ppm}$ to $u_{\mathrm{r}}\left(k_{\mathrm{B}}\right)$. Present-day acoustic apparatus acquires data fully automatically; therefore, it is easy to acquire five or ten times the amount of data that was acquired in 1988. This will significantly reduce the statistical uncertainties, provided that the replicated data are statistically independent.

In [3] the temperature was measured by three capsule-type standard platinum resistance thermometers (SPRTs) that were calibrated before and after the acoustics measurements. Consequently, the largest component of the temperature uncertainty resulted from the random uncertainty of the calibrations and it contributed $0.8 \mathrm{ppm}$ to $u_{\mathrm{r}}\left(k_{\mathrm{B}}\right)$. This component of the uncertainty could be reduced by a factor of 5 or more by adopting the procedures used in primary thermometry at high-temperatures. $[9,10]$ To do so, one or more thermal wells must be built into the acoustic apparatus. Then, thermometers can be transferred between these wells and triplepoint-of-water cells.

\section{Volume Determinations}

\subsection{Pycnometry}

In the 1988 re-determination of $k_{\mathrm{B}}$, the volume of the spherical cavity was determined by weighing the mercury required to fill the cavity at $T_{\mathrm{TPW}}$ and using literature values for the density of the particular sample of mercury used. [3] The relative uncertainty of the volume was $u_{\mathrm{r}}(V)=$ $1.2 \mathrm{ppm}$. Because the value of $k_{\mathrm{B}}$ depends on $V^{2 / 3}$, these measurements contributed $0.80 \mathrm{ppm}$ to $u_{\mathrm{r}}\left(k_{\mathrm{B}}\right)$ and the largest contribution to this uncertainty $(0.67 \mathrm{ppm})$ came from literature values of the thermal expansion of mercury in the range $0{ }^{\circ} \mathrm{C}$ to $20^{\circ} \mathrm{C}$. If pycnometry were used today, the volume of the cavity would be determined near $20{ }^{\circ} \mathrm{C}$ using a particular sample of a liquid (e.g. water, mercury, tridecane) whose density was determined at $20{ }^{\circ} \mathrm{C}$, probably by using one of Cook's techniques. [38] Then, the thermal contraction of the cavity between $20^{\circ} \mathrm{C}$ and $T_{\mathrm{TPW}}$ would be determined with a much smaller uncertainty from measurements of the temperaturedependence of the resonance frequencies of the microwave modes within the same cavity. If this 
were done and if the uncertainties achieved in [3] were reproduced, the uncertainty of $V^{2 / 3}$ would be reduced to $0.43 \mathrm{ppm}$.

Pycnometry is usually conducted at ambient pressure. The volume of the acoustic cavity is needed either at the pressures at which the acoustic frequencies are measured or at zero pressure, depending upon how the data are analyzed. When hydrostatic pressure is applied to a cavity bounded by isotropic steel walls, its volume will shrink by the fraction $6 \times 10^{-6}(\mathrm{p} / \mathrm{MPa})$.

\subsection{Microwave Resonances.}

The quasi-spherical cavity was invented to simplify the determination of the thermal expansion of acoustic cavities used for primary thermometry. [13] As reported elsewhere in this volume, significant effort is being devoted to determining the volume of a quasi-spherical cavity from microwave measurements.

The microwave resonances in a perfectly spherical cavity occur in overlapping multiplets that have degeneracies of $(2 l+1)$ where $l=1,2,3, \ldots$ Unavoidable imperfections in construction partially remove the degeneracies, thereby creating the difficult measurement problem of determining the average value of three or more overlapping resonances, particularly when the degree of overlap is temperature-dependent, either because of temperature-dependent conductivity of the cavity's walls or because the thermal expansion of the cavity is anisotropic. The quasi-spherical cavity is designed to have sufficiently large deviations from a perfect sphere to fully separate the components of the triply-degenerate modes. A numerically controlled milling machine can manufacture a "hemispherical" shells with inner surfaces approximating a triaxial ellipsoid with axes of lengths $a, a\left(1+e_{1}\right)$, and $a\left(1+e_{2}\right)$ where $e_{1}$ and $e_{2}$ are small numbers. For example, if $e_{1} \approx 0.002$ and $e_{2} \approx 0.001$, the microwave triplets that would be degenerate in a perfect copper sphere with $a=5 \mathrm{~cm}$ are split by more than 5 times their half-widths at $T_{\mathrm{TPW}}$; if the quasi-sphere were stainless steel, the splitting would be 1.5 times the half-widths. With this splitting, the average frequency of a multiplet can be determined with a relative uncertainty of $10^{-8}$ or less. This is sufficient to measure the thermal expansion of the quasi-sphere with an uncertainty of $10^{-8}$ or less. For the same values of $e_{1}$ and $e_{2}$, the perturbations to the acoustic frequencies are calculable and they are small compared with the separation between the closely spaced $(0,2)$ and $(3,1)$ acoustic modes.

To determine $V^{2 / 3}$ with a relative uncertainty of $1 \mathrm{ppm}$ or less from microwave measurements, one requires a theoretical relationship between the measured frequencies and the volume that is correct to order $e_{1}{ }^{2}$ and $e_{2}{ }^{2}$. Recently, Mehl [39] developed the necessary theory and showed that it that correlates certain microwave data at the $1 \mathrm{ppm}$ level. Now, systematic studies are required to account for the coupling of the microwave modes of the cavity to the antennas, coaxial cables, and measuring instruments. These studies would be the microwave equivalent of the studies of microphones conducted by Guianvarc'h et al. [15] The microwave method has the advantage that many microwave triplets, perhaps spanning a factor of 10 in frequency, can be used to determine the same volume. Thus, there are many consistency checks. A weakness of this method is that there is no independent way to measure the conductivity of the cavity's walls at microwave frequencies; thus, the measured widths of the microwave modes cannot be used to critically test the theory. (In contrast, the widths of the radially symmetric acoustic modes in a gas-filled spherical cavity can be compared with independently-determined transport properties of the gas.)

\subsection{Dimensional Metrology.}


As reported elsewhere [40], significant effort is being devoted to determining the volume of a cavity from dimensional measurements. From the perspective of a non-expert, there are two parts to this problem: (1) determine the shape of a hemispherical (or quasi-hemispherical) cavity from dimensional measurements, and (2) determine from exterior measurements, how the operations of assembling two hemispherical cavities into a single spherical cavity influence dimensions of the cavity. Problem (1) has been carefully explored by comparing the coordinates of either a silicon or a zerodur sphere to the coordinates of hemispherical cavities using the same coordinate measuring machine at the same time. Problem (2) is somewhat analogous to the dimensional changes that occur when "wringing" two gage blocks together. The wringing of gauge blocks has a long history. [41] During a recent Key Comparison [42], 11 laboratories wrung together pairs of gage blocks and measured the resulting lengths. The standard deviation of the repeatability of the lengths ranged from 3 to $12 \mathrm{~nm}$, depending upon the laboratory. This suggests that two hemispherical shells could be assembled into a spherical shell with a relative volume uncertainty of only $10 \mathrm{~nm} / a$, where $a$ is the radius. However, achieving this remarkable performance might require the mating surfaces of the hemispheres to be as hard and as flat as the ends of gage blocks.

\section{Advances in cylindrical resonators.}

In cooperation with NIST, Zhang et al. [43] at the Chinese National Institute of Metrology (NIM) are actively studying the longitudinal modes $\left(f_{\mathrm{n}} \approx n u L / 2\right)$ of gas-filled, cylindrical cavities of length $L$ for re-determining $k_{\mathrm{B}}$. The spirit of their work follows the pioneering acoustic determination of $k_{\mathrm{B}}$ of Quinn et al. [1]; however, there are numerous innovations. The longitudinal modes are non-degenerate and their resonance frequencies are not sensitive, in the first order of perturbation theory, to misalignments of the ends of the cavity. The prototype

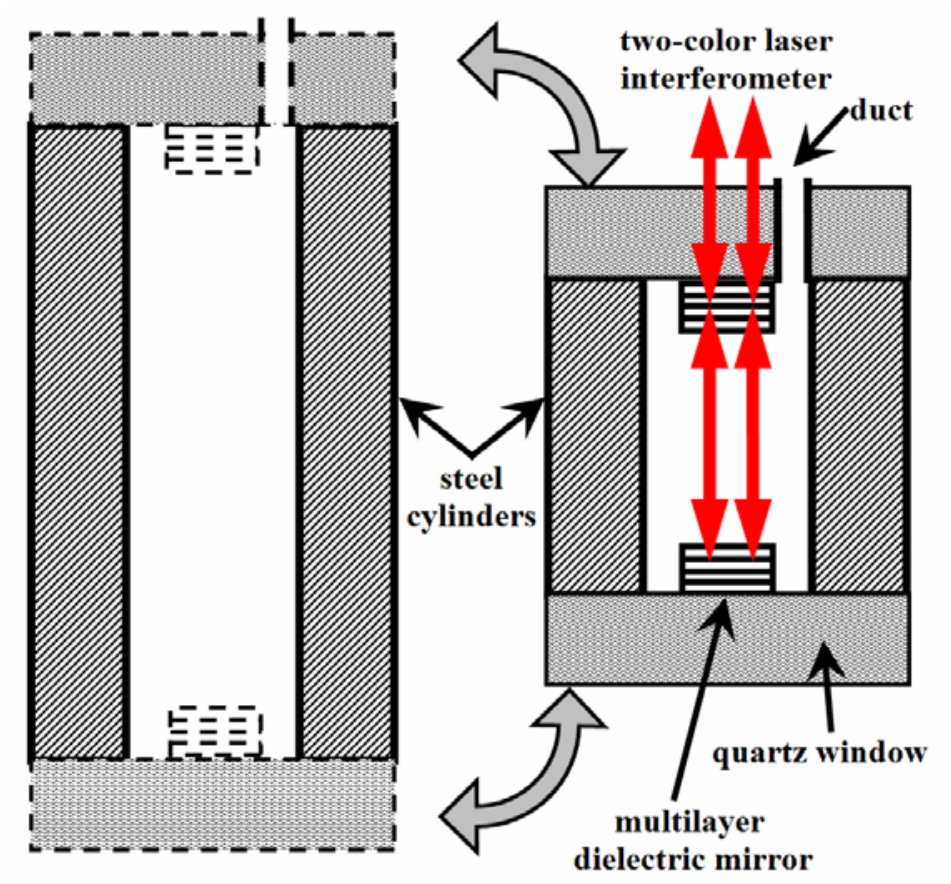

Fig. 4 Schematic diagram of NIM acoustic/optical interferometer. [43] 
resonator had an inside radius $a=50 \mathrm{~mm}$; therefore, the quality factors (Qs) of the longitudinal modes were approximately double those obtained by Quinn et al in their cylinder with $a=$ $15 \mathrm{~mm}$. For a cylinder of length $L=2 a$, the $Q$ s of the lowest few longitudinal modes are approximately $1 / 5$ of the $Q$ s of the lowest few radially symmetric modes of a spherical cavity with a radius $a$; this is a disadvantage. However, the $Q$ s of the cylinder's modes are large enough that small transducers can be used to excite and detect the modes while generating only small perturbations to the resonance frequencies.

Quinn et al. used a movable piston to continuously vary the length of the resonator. In contrast, the NIM group will use two cylindrical cavities with fixed lengths in the ratio 2:1. (See Fig. 4.) They will transfer a single pair of cavity ends from one cylinder to the other. The transfer of the ends avoids problems resulting from the gap or seals between a moveable piston and the cylinder enclosing it. The 2:1 length ratio facilitates an approximate cancellation of perturbations from the acoustic admittances of the cavities' ends. Neglecting perturbations, the even longitudinal modes $(n=2,4,6, \ldots)$ of the longer cavity will have the same resonance frequencies as the longitudinal modes of the shorter cavity. The frequencies of these modes are perturbed by the frequency- and position-dependent specific acoustic admittances $\beta(\boldsymbol{r}, f)$ of the surfaces of the cavities. The surface perturbations $\Delta \boldsymbol{F}_{\text {surface }}$ can be calculated from the sum of a surface integral over the ends of the cavity and another surface integral over the cylindrical sides of the cavity:

$$
\Delta \boldsymbol{F}_{\text {surface }} \equiv \Delta f_{\text {surface }}+i g_{\text {surface }} \approx \frac{i u}{4 \pi}\left[\int_{\text {ends }} \beta(r, f) \phi_{\mathrm{n}}^{2}(r) d s+\int_{\text {sides }} \beta(r, f) \phi_{\mathrm{n}}^{2}(r) d s+\right] / \int_{\text {volume }} \phi_{\mathrm{n}}^{2}(r) d V .
$$

(Here $\phi_{\mathrm{n}}$ is the velocity potential.) Because both resonators operate at the same frequencies and use the same ends, the integrals over the ends $\int_{\text {ends }}$ are identical for both resonators and independent of $L$. However, the volume integral $\int_{\text {volume }}$ and the integral over the sides $\int_{\text {sides }}$ are proportional to $L$. Therefore, results from both resonators can be combined to eliminate $\int_{\text {ends }}$ from the calculation of $k_{\mathrm{B}}$. The eliminated contributions originate in the thermo-acoustic boundary layer on the multilayer dielectric mirrors and in ducts and/or acoustic transducers embedded in the ends. If the thermal accommodation lengths $l_{\mathrm{a}}$ at the mirrors do not change when the ends are transferred from one cylinder to the other, their contributions to $k_{\mathrm{B}}$ and $\mathrm{u}\left(k_{\mathrm{B}}\right)$ will also be eliminated.

When the effects of $\delta_{\mathrm{T}}$ and $\delta_{\text {viscosity }}$ are considered, the acoustical lengths of the resonators will differ from their optically-measured lengths by pressure-dependent terms of order $O\left(\delta_{\Gamma} / L\right) \sim$ $2 \times 10^{-3}$. Therefore, the resonance frequencies of the two resonators will not be identical and the cancellation of the perturbations from the ends will not be exact.

The NIM group will use a two-color laser interferometer to determine the distance between the multilayer dielectric mirrors on the inner surface of each end of the cavity. This optical length depends upon optical phase shifts within the dielectric mirrors and on the thicknesses of the mirrors. However, the difference between the optical lengths of the two resonators will be independent of the mirrors because the same mirrors will be used for both resonators.

To summarize, the two-cylinder approach to an acoustic measurement of $k_{\mathrm{B}}$ will use an optical method to measure the distances between the inside surfaces of the ends of two cylindrical cavities after the cavities are assembled. In contrast, the dimensional metrology of the interior surfaces of two hemispheres (Section 7.3) must be conducted before the hemispheres are assembled to for a cavity. The two-cylinder approach avoids the complexities of pycnometry and it does not require advances in the understanding of microwave cavities. However, the 
lower Qs of the acoustic resonances in the cylindrical cavities (compared with spherical cavities) will complicate the measurement and the interpretation of the acoustic resonance frequencies.

\section{Concluding Remarks}

We have reviewed the advances made since 1988 in understanding acoustic resonators. Stimulated by primary acoustic thermometry, the uncertainties from every aspect of the acoustic measurements have been understood and can be smaller than they were in 1988 . The uncertainties of the transport properties of helium and argon have been reduced by approximately a factor of 10 . If thermometer wells are built into an acoustic apparatus, $T_{\mathrm{TPW}}$ can be achieved in the apparatus with a much smaller uncertainty than was achieved in 1988. With this progress, one could re-determine $k_{\mathrm{B}}$ today from acoustic measurements using either helium or argon with a relative uncertainty $u_{\mathrm{r}}\left(k_{\mathrm{B}}\right) \approx 10^{-6}$. A further reduction in uncertainty is possible if the acoustic volume of a spherical cavity can be determined more accurately than it was in 1988 . Several groups are actively researching ways to do this. If argon is used to re-determine $k_{\mathrm{B}}$ with $u_{\mathrm{r}}\left(k_{\mathrm{B}}\right)<10^{-6}$ the relative abundances of the argon isotopes and noble gas impurities must be determined more accurately than they were in 1988.

\section{Acknowledgements}

I thank R. Davis, R. Gavioso, K. Gillis, K. Hill, J. Mehl, M. McLinden, L. Pitre, and D. Ripple for suggesting improvements to this article. I thank R. Berg, R. Gavioso, K. Gillis, H. Lin, J. Mehl, L. Pitre, D. Ripple, and J. Zhang for sharing unpublished results with me.

\section{References:}

[1] A. R. Colclough, T. J. Quinn, and T. R. D. Chandler, Proc. R. Soc. London Ser. A 368, 125 (1979); T. J. Quinn, A. R. Colclough, and T. R. D. Chandler, Philos. Trans. R. Soc. London, Ser. A 283, 367 (1976).

[2] E. R. Cohen and B. N. Taylor, Rev. Mod. Phys. 59, 1121 (1987).

[3] M. R. Moldover, J. P. M. Trusler, T. J. Edwards, J. B. Mehl, and R. S. Davis, J. of Res. of NBS, 93, 85 (1988).

[4] P. J. Mohr and B. N. Taylor, Rev. Mod. Phys. 72, 351 (2000); P. J. Mohr, B. N. Taylor, and D. B. Newell, Rev. Mod. Phys. 80, 633 (2008).

[5] M. R. Moldover, S. J. Boyes, C. W. Meyer, and A. R. H. Goodwin, J. Res. Natl. Inst. Standards Technol. 104, 11 (1999).

[6] M. B. Ewing and J. P. M. Trusler, J. Chem. Thermodynam. 32, 1229 (2000).

[7] G. Benedetto, R. M. Gavioso, R. Spagnolo, P. Marcarino and A. Merlone, Metrologia 41, 74 (2004).

[8] Pitre, L., Moldover, M. R. and Tew, W. L., Metrologia, 43, 142 (2006).

[9] D. C. Ripple, G. F. Strouse, and M. R. Moldover, Int. J. Thermophysics 28, 1789 (2007).

[10] D. C. Ripple, D. R. Defibaugh, M. R. Moldover, and G. F. Strouse, in “TEMPERATURE: Its Measurement and Control in Science and Industry; Volume VII; 8th International Temperature Symposium, Chicago IL, Oct. 21-24, 2002,” edited by D.C. Ripple, pp. 25-30, A. I. P. Conf. Proc. (2003).

[11] A. R. H. Goodwin, “Thermophysical Properties from the Speed of Sound,” Ph. D. Thesis, University College London (1988) pp. 113-117. 
[12] M. B. Ewing, J. B. Mehl, M. R. Moldover, and J. P. M. Trusler, Metrologia 25, 211 (1988).

[13] J. B. Mehl, M. R. Moldover, and L. Pitre, Metrologia 41, 295 (2004).

[14] J. B. Mehl and M. R. Moldover, private communication.

[15] C. Guianvarc'h, R. Gavioso, G. Benedetto, L. Pitre, and M. Bruneau, submitted to J. Appl. Phys. (2009).

[16] M. Greenspan, J. Acoustical Society of America, 28, 644 (1956).

[17] J. J. Hurly and J. B. Mehl, J. Res. Natl. Inst. Stand. Technol. 112, 75 (2007).

[18] E. Bich, R. Hellmann and E. Vogel, Molecular Phys. 105, 3035 (2007).

[19] E. F. May, M. R. Moldover, R. F. Berg, and J. J. Hurly, Metrologia 43, 247 (2006).

[20] J. Kestin and W. Leidenfrost Physica 251033 (1959).

[21] C. Evers, H. W. Lösch, and W. Wagner, Int. J. Thermophys. 23, 1411 (2002).

[22] R. F. Berg Metrologia, 4211 (2005); R. F. Berg, Metrologia 43, 183 (2006) (Erratum); R.F. Berg, Private Communication.

[23] J. J. Hurly and M. R. Moldover, J. Res. Nat. Inst. Standards Technol. 105, 667 (2000).

[24] M. Jeziorska, W. Cencek, K. Patkowski, B. Jeziorski, and K. Szalewicz, J. Chem. Phys. 127, 124303 (2007).

[25] J. B. Mehl, Comptes Rendus Physique, 10859 (2009).

[26] P. E. Suetin, B. T. Porodnov, V. G. Chernjak and S. F. Borisov, J. Fluid Mech. 60, 581 (1973).

[27] J. B. Mehl, J. Acoust. Soc. Am. 78, 782 (1985).

[28] L. Pitre, private communication.

[29] R. M. Gavioso, G. Benedetto, P. A. Giuliano Albo, INRIM Thermodynamics Div., unpublished report (2006).

[30] J. B. Mehl and M. R. Moldover unpublished.

[31] M. B. Ewing, M. L. McGlashan, and J. P. M. Trusler, Metrologia 22, 93 (1986).

[32] Kennard, E. H., “Kinetic Theory of Gases,” (McGraw-Hill, New York, 1938), pp. 311-316.

[33] K. A. Gillis, I. I. Shinder, and M. R. Moldover, Phys. Rev. E 70, 021201 (2004).

[34] J. B. Mehl, unpublished.

[35] D. C. Ripple, D. R. Defibaugh, K. A. Gillis, and M. R. Moldover, Proceedings of TEMPMEKO '99, J. Dubbeldam and M. de Groot, eds., p. 418 (1999).

[36] D. C. Ripple, private communication.

[37] H. Lin, K.A. Gillis. M. R. Moldover, J. Res. Natl. Inst. Standards Technol. 114, 263 (2009).

[38] Cook, A. H., and Stone, N. W. B., Trans. R. Soc. London A 250, 279 (1957); Cook, A. H., Phil. Trans. R. Soc. London A 254, 125 (1961).

[39] J. Mehl, Metrologia 46, 554 (2009).

[40] Comptes Rendus, this issue.

[41] G. J. Siddall and P. C. T. Willey, J. Phys. D. 3, 8 (1970).

[42] R. Thalmann, Metrologia 39, 165 (2002).

[43] J. T. Zhang, H. Lin, Y. Y. Duan, and X. J. Feng, Acta Metrologica Sinica 29, 1 (2008). 